Voix et Images

voixetimages

\title{
Une dramaturgie (con)cernée par les médias
}

\section{Hervé Guay et Francis Ducharme}

Volume 39, numéro 1 (115), automne 2013

Théâtre et médias

URI : https://id.erudit.org/iderudit/1022989ar

DOI : https://doi.org/10.7202/1022989ar

Aller au sommaire du numéro

Éditeur(s)

Université du Québec à Montréal

ISSN

0318-9201 (imprimé)

1705-933X (numérique)

Découvrir la revue

Citer ce document

Guay, H. \& Ducharme, F. (2013). Une dramaturgie (con)cernée par les médias .

Voix et Images, 39(1), 7-15. https://doi.org/10.7202/1022989ar d'utilisation que vous pouvez consulter en ligne.

https://apropos.erudit.org/fr/usagers/politique-dutilisation/ 


\title{
UNE DRAMATURGIE (CON)CERNÉE PAR LES MÉDIAS 1
}

\author{
$+++$ \\ HERVÉ GUAY \\ Université du Québec à Trois-Rivières \\ FRANCIS DUCHARME \\ Université du Québec à Montréal
}

Le danger pour l'Amérique, ce ne sont pas les Pensées de Mao:

$c^{\prime}$ est la prolifération des caméras ${ }^{2}$.

Tumultueuse, la relation entre le thêâtre et les médias l'a toujours été au Québec, depuis ses débuts il y a près de deux cent cinquante ans. Mais les raisons de ce tumulte ont bien changé. Alors que, durant une bonne partie du XIXe et même du XXe siècle, la presse était divisée quant aux mérites du théâtre et au bien-fondé de sa participation aux débats sociétaux, ce sont plutôt les auteurs dramatiques qui débattent aujourd'hui le rôle joué par les médias dans l'espace public et la façon dont ceux-ci le façonnent. Que le thêâtre québécois se préoccupe autant des médias, pendant que ces derniers se désintéressent de lui, nous paraît révélateur à la fois de ce que la dramaturgie et les médias sont devenus. C'est ce qui nous a poussés à consacrer un dossier au théâtre et aux médias, à nous intéresser à ce que le théâtre pouvait dire de l'évolution de ces derniers. Il convient, à nos yeux, d'examiner la critique que cet art désormais minoritaire véhicule d'un système sinon omnipotent, du moins tentaculaire. Qui aurait d'ailleurs pensé que des vues si partiales sur la culture médiatique verraient le jour dans le giron du monde du spectacle à partir duquel Guy Debord avait formé son célèbre syntagme pour stigmatiser les dérives de la société de consommation et de divertissement?

Régis Debray en convient: les dérivés du mot medius ont pris une extension démesurée ${ }^{3}$. Mais est-il si sûr, comme il le prétend, que la médiation soit «multiséculaire, propre à l'espèce - transhistorique ${ }^{4} »$ ? Cela ne dépend-il pas de l'acception que l'on donne au mot «média »? La notion, ainsi que sa racine latine l'indique (centre,

$$
++
$$

1 La réalisation de ce dossier a été rendue possible grâce au financement du projet «La mise à distance du spectateur dans le théâtre québécois contemporain » par le Fonds de recherche du Québec - Société et culture (FRQSC). 2 Jean Genet, Fuvres complètes, t. VI: L'ennemi déclaré. Textes et entretiens, édition établie et annotée par Albert Dichy, Paris, Gallimard, coll. «Nouvelle revue française», 1991, p. 318.3 "Ouvrez le mot médio de médiologie, et, d'où que vous veniez, vous découvrirez une poupée russe: du dehors vers le dedans, les médias, le médium, le médian, le médiat. Une lettre change, l'accent se déplace, chaque cran s'imbrique dans le précédent. La médiologie comme le final d'un travail de désemboîtement progressif. » Régis Debray, "Histoire des 4M", Les cahiers de médiologie, Paris, Centre national de la recherche scientifique (CNRS), 2009, p. 13.4 Ibid. 
milieu), renvoie à la rencontre entre deux points. C'est ce sens qui a mené à la création du terme français «intermédiaire», repris aujourd'hui dans l'emploi du mot «média » pour désigner un moyen de communication. Cette idée de moyen, elle-même équivoque, a d'abord servi à désigner des instruments, outils et techniques de communication. C'est dans ce sens qu'on l'utilise, par exemple, dans les études du médiatique au thêâtre, qui sont de plus en plus nombreuses, les échanges formels entre les deux secteurs étant extrêmement féconds. L'analyse des interactions entre ces diverses formes d'expression a même donné naissance à un nouveau champ du savoir, les études intermédiales, auxquelles participent quantité de chercheurs réunis notamment autour de la revue Intermédialités. Comme ce va-et-vient retient déjà l'attention de nombreux chercheurs, il en sera peu question dans ce dossier consacré à la représentation des médias dans la dramaturgie québécoise.

Or, ces dernières décennies, les principaux moyens de télécommunication et de communication ont fortement accru leur importance institutionnelle, au point qu'un consortium du secteur du journalisme ou des communications peut maintenant être désigné couramment comme un média - plutôt que comme un médium, l'accusatif conservé du latin étant généralement réservé aujourd'hui pour désigner un moyen d'expression artistique. En anglais, on dénomme mass media ces mastodontes qui canalisent aujourd'hui l'essentiel des échanges communicationnels. Il sera donc question ici des médias de masse en tant qu'institution économique, politique, culturelle et discursive pour éviter d'étendre la problématique à l'infini et ainsi privilégier l'ancrage contemporain de ce dossier. En partie liée à cette acception du terme, la notion de culture médiatique est également centrale, car elle prend justement en considération le pouvoir de l'institution d'orienter les comportements et les manières de penser des individus - pour le meilleur et pour le pire.

L'une des raisons expliquant la préoccupation croissante du théâtre à l'égard $\mathrm{du}$ «quatrième pouvoir» est sans doute l'évolution contraire des deux moyens de communication. Alors que le théâtre a été, des siècles durant, au cœur de la culture occidentale, s'imposant même un temps comme l'un des vecteurs de la culture populaire, il a dû progressivement céder du terrain sous la pression cumulée de la presse, du cinéma, de la radio, de la télévision, d'Internet et du numérique, moyens de diffusion d'une puissance supérieure à celle des salles de spectacle de la plupart des grandes villes. La massification des médias, commencée avec la presse et poursuivie par Internet, n'en finit plus de s'accroître et la mondialisation des échanges ne fait qu'accélérer le mouvement. D'où le repositionnement du thêâtre dans le champ culturel par rapport aux grands médias constaté par Isabelle Barbéris : «le champ du spectacle vivant au $\mathrm{XX}^{\mathrm{e}}$ siècle s'est construit comme contrechamp des industries culturelles et du marché de $l^{\prime} a^{5}{ }^{5}$ ». Incapable de concurrencer les médias à cause de son modèle économique, la scène est devenue, selon elle, un secteur "archaïque» dont la crise «intensifie la fonction critique et sert d'argument nécessaire ${ }^{6}$ » à sa survie. Si la situation des arts de la scène au Québec ne ressemble pas en tous points à celle de la France

$$
++
$$

5 Isabelle Barbéris, Théâtres contemporains. Mythes et idéologies, Paris, Presses universitaires de France, coll. «Intervention philosophique», 2010, p. 10. 6 Ibid., p. 11. 
d'où parle Barbéris, nous observons, tout comme elle, une résistance manifeste de la dramaturgie québécoise à l'emprise de la culture médiatique.

En clair, les médias constituent dorénavant un centre d'intérêt, voire une cible privilégiée des auteurs dramatiques. Tel n'a pas toujours été le cas. On chercherait en vain dans le théâtre de Gratien Gélinas, de Marcel Dubé ou de Pierre Perrault une pièce où ils occupent le devant de la scène. Fini le temps où un reporter pouvait encore être vu comme un agent positif de changement grâce à son rôle dans la lutte contre la corruption et l'incompétence (Le reporter ${ }^{7} \mathrm{~d}^{\prime}$ Alexandre Huot), où la radio devenait un gage d'ouverture sur le monde (C'était avant la guerre à l'Anse-à-Gilles ${ }^{8}$ de Marie Laberge). Mais voilà : les médias ne sont plus ce qu'ils étaient. Ainsi semblent en avoir décidé les auteurs dramatiques d'aujourd'hui. Ils estiment que les symptômes de cette détérioration sont partout: dans la qualité vacillante de l'information et de la fiction; dans l'omniprésence de la publicité; dans le populisme et le sensationnalisme; dans les effets déstabilisants des nouvelles technologies sur les habitudes de lecture et d'écoute, sur le travail culturel et sur les droits d'auteur; dans la dématérialisation de la réalité sensible, etc. Plus rarement le dramaturge se montre-t-il optimiste à l'égard de l'évolution du monde des communications. Cette critique des médias par le théâtre s'inscrit a fortiori dans un contexte de concentration et de convergence inégalées: de vastes empires se forment par le biais de l'acquisition de très nombreuses entreprises ainsi que d'autres maillons de la chaîne de production et de diffusion de la culture et des communications (producteurs, éditeurs, imprimeurs, libraires, services de téléphonie et d'Internet...). Ces géants influent donc de manière accrue sur des aspects multiples de la vie sociale et culturelle ${ }^{9}$. La consolidation d'un oligopole mondial et multisectoriel, pour reprendre les mots de Robert McChesney et Noam Chomsky ${ }^{10}$, accentue l'urgence d'une réflexion sur l'état des institutions culturelles et démocratiques. Comme les travailleurs du milieu culturel et les auteurs dramatiques évoluent eux aussi dans cette culture médiatique, il n'est pas étonnant qu'ils soient si préoccupés par une situation qui les touche de près sur le plan intellectuel comme sur le plan économique.

Compte tenu de la variété des tribunes, des modes d'intervention et des formes employées dans le monde des communications, le regard porté sur ce système par le théâtre québécois cible des supports et des acteurs multiples, en plus de recourir à des tonalités et à des angles d'attaque variés. L'on peut tout de même risquer une tentative de catégorisation des secteurs où se déploie cette critique dans les œuvres auxquelles les collaborateurs de ce dossier se sont intéressés.

La mission d'information confiée aux médias de masse fait réagir vivement les auteurs dramatiques québécois contemporains. Parce que les grands médias réservent

$$
+++
$$

7 Alexandre Huot, Le reporter. Comédie hérö̈que, Montréal, Éditions Édouard Garand, coll. «Théâtre canadien», 1930, 36 p. 8 Marie Laberge, C'était avant la guerre à l'Anse-à-Gilles, Montréal, VLB, 1981, 119 p. 9 Ce phénomène provient de la vague de déréglementation amorcée aux États-Unis au cours des années 1980. Au Québec, cette convergence s'est accélérée particulièrement depuis le début des années 2000. Voir Anne-Marie Gingras, "La concentration de la propriété de la presse», Médias et démocratie. Le grand malentendu, Québec, Presses de l’Université du Québec, 2009 [1999], p. 132-163. 10 Robert W. McChesney, «La consolidation de l'oligopole des médias », Noam Chomsky et Robert W. McChesney, Propagande, médias et démocratie, Montréal, Écosociété, 2000, p. 101-109. 
une place limitée à l'information, ce mandat ne paraît pas rempli de manière satisfaisante à leurs yeux. À l'instar d'Anne-Marie Gingras, ils sont plusieurs à croire qu'il y a un malentendu persistant entre l'idéal du journalisme hérité des Lumières (les médias comme sphère publique neutre, comme quatrième pouvoir indépendant, transparent, rationnel théorisé par Jürgen Habermas) et sa réalisation concrète perçue comme lacunaire et faussée par le système économique. De là à souscrire à la critique de l'auteure de Médias et démocratie, selon laquelle les médias sont en fait beaucoup plus proches de l'appareil idéologique au service du marché, sinon de l'État, il n'y a qu'un pas que bien des auteurs franchissent. L'invention du journalisme a beau avoir contribué à rendre possibles nos démocraties libérales, son histoire est aussi liée à l'essor de la publicité et du capitalisme, dont les grands magnats de la presse sont devenus des figures emblématiques. D'aucuns conviennent que, si les médias concourent à la formation de l'espace public - ce que la notion même de publicité désignait avant qu'elle ne remplace le vieux mot de réclame - la communauté ainsi assemblée, comme l'ont constaté plusieurs sociologues dès l'avènement de la télévision, a sans doute plus à voir avec la civilisation des loisirs espérée par Joffre Dumazedier ${ }^{11}$ et la société de consommation analysée par David Riesman ${ }^{12}$ qu'avec l'agora politique propice aux débats prétendument caractéristiques de la démocratie.

L'information culturelle s'avère un autre sujet de préoccupation des dramaturges québécois. Étant donné le rôle de la réception critique dans la reconnaissance de la littérature et du thêâtre, il n'y a là rien d'étonnant. Comme les artistes sont directement touchés par la qualité du travail effectué par les journalistes affectés à la culture, il est normal que cette fonction des médias ait été l'une des premières à faire l'objet de commentaires de la part de ceux et celles qui écrivent pour la scène. Il ne faut pas alors s'attendre, ainsi que nous l'enseigne l'exemple de L'impromptu de l'Alma d'Eugène Ionesco, à ce que les artistes fassent preuve de plus d'aménité que les critiques en ont eu à leur égard dans le portrait qu'ils brossent d'une profession que personne n'aime ${ }^{13}$. À moins bien sûr que l'écrivain ne soit aussi critique et que ce dernier constitue un double de l'auteur dans l'un de ses poèmes dramatiques. En outre, on pense peut-être moins, quand les écrivains font le procès de la critique, à la conflagration entre culture populaire et culture savante, source de divergence fréquente entre les deux parties. Certains auteurs dramatiques s'efforcent donc, par divers moyens, d'éviter que leur critique ne soit perçue comme une attaque élitiste contre la culture populaire, d'autant plus qu'il semble devenu impossible de se retrancher hors de portée des médias. Quoi qu'il en soit, la problématique de l'autorité en matière d'opinion culturelle, le choix des critères d'évaluation des œuvres, la personnalité qui les applique, la tonalité dans laquelle cela se fait appartiennent aussi à l'équation, de même que la participation ou non de l'artiste à la commercialisation de l'art.

11 Joffre Dumazedier, Vers une civilisation du loisir?, Paris, Éditions du Seuil, coll. «Esprit», 1962,318 p. 12 David Riesman, La foule solitaire. Anatomie de la société moderne, préface d’Edgar Morin, Paris, Arthaud, 1964 [1950], 378 p. 13 Georges Banu, «Préface», Hervé Guay (dir.), Franchir le mur des langues, Montréal, Éditions du Canal, 2005, p. ix. 
Le contenu des médias - «la programmation» comme on le dit pour la radio et la télévision, «l'espace rédactionnel» si on reprend le terme employé dans la presse écrite - n'échappe pas non plus à la vigilance des auteurs dramatiques. L'observateur le moins perspicace remarque déjà la faible proportion qu'occupent les reportages et les documentaires dans la grille horaire de la télévision comparativement aux jeux et à la fiction. Ainsi, même à la radio publique, beaucoup de temps d'antenne est consacré à la musique et à l'humour. Seule la presse écrite accorderait encore la part du lion au journalisme et résisterait à la prédominance du divertissement. Or, même le journalisme "papier», plutôt situé dans le champ de production restreinte, subit l'influence institutionnelle de la télévision, située davantage dans le champ commercial. Celle-ci exerce sur lui une pression pour qu'il se conforme à son style, à ses orientations ${ }^{14}$. C'est sans compter la concurrence d'Internet et du numérique et leur rôle crucial dans la circulation de l'information, de la fiction et d'une multitude de jeux et de divertissements de tout acabit. Cette influence se fait sentir notamment dans le développement récent par La Presse d'une plateforme numérique.

Le contenu des médias est généralement abordé par les auteurs dramatiques selon deux angles: celui des habitudes de fréquentation et des comportements induits par les pratiques médiatiques et celui des esthétiques privilégiées dans un média donné. Sur le plan dramaturgique, il en résulte diverses émanations de l'homo mediaticus, qu'il soit agent ou sujet de cette médiatisation, généré, traversé ou annihilé par elle. Mais le choc des écritures dramatiques et médiatiques ne manque pas de produire juxtaposition, opposition, friction, hybridation, parodie et satire. La tentation de l'intermédialité apparaît forcément, puisque la thématisation des médias dans l'écriture ne saurait être seulement abstraite : elle appelle aussi sa contrepartie scénique, la présence physique des outils de communication sur le plateau, auxquels la mise en scène accordera un espace plus ou moins grand selon les œuvres ${ }^{15}$.

Parallèlement, les études télévisuelles relèvent une uniformisation des contenus. Michèle Martin et Serge Proulx remarquent le mélange croissant du divertissement et de l'information: "Que ce soit pour les émissions d'affaires publiques, d'information, d'éducation ou autres, on veut attirer les publics en leur donnant l'impression qu' on les divertit ${ }^{16}$. » Cette fusion ou plutôt cette confusion des genres entraîne, on le comprend, un virage généralisé vers le divertissement, qui a toujours été une visée des émissions médiatiques et des genres journalistiques, mais sans que celle-ci domine aussi clairement les intentions de ces multiples discours et fasse de leurs destinataires des êtres en perpétuelle quête d'amusement.

14 Pierre Bourdieu, Sur la télévision suivi de L'emprise du journalisme, Paris, Raisons d’agir, 1996, p. 62. 15 Signalons le paradoxe : si les médias sont sévèrement critiqués, les emprunts formels du théâtre à leur endroit n’ont jamais été si nombreux et si utiles à leur critique. La démystification du fonctionnement médiatique passe en effet moins par sa représentation directe que par celles des coulisses où se fabrique la médiatisation avant d'en arriver au produit ou au montage final. 16 Michèle Martin et Serge Proulx, Une télévision mise aux enchères. Programmation, programmes, publics, Sainte-Foy, Télé-université, coll. «Communication et société», 1995, p. 73. Les auteurs citent Gaëtan Tremblay et Roch Harvey, «Évolution des stratégies de programmation des radiodiffuseurs conventionnels», Communication, vol. XII, n² 2, 1991, p. 53-79. 
Bien que le théâtre n'ait pas été épargné par l'hybridité et l'hétéromorphie, deux phénomènes scrutés dans les études thêâtrales depuis au moins une trentaine d'années, l'influence du roman, de la poésie et de l'essai (hybridité), tout comme celle du cinéma, de la télévision, de la danse, de l'installation et de la performance (hétéromorphie) n'ont pas entraîné beaucoup de reconnaissance de l'influence réelle des médias sur le renouvellement esthétique du théâtre, ni suscité une véritable interrogation au sujet de son insertion dans la «société du spectacle». Dans leur grande majorité, les auteurs dramatiques contemporains ont plutôt voulu se distancier de la logique de pur divertissement qu'ils associent aux médias, faisant peu état de leurs éventuels effets positifs (enquête sur des problèmes sociaux, combat pour une cause, mise en scène de soi, liberté d'expression, ouverture sur le monde, etc.). Depuis 2000, au moins quatre dossiers de la revue Jeu ${ }^{17}$ ont signalé le retour de l'engagement au théâtre, c'est-à-dire le mélange parfois périlleux de deux intentions, l'une esthétique, l'autre sociopolitique. Or, si les problèmes de la cité abordés au théâtre sont pluriels, la prise à partie des médias de masse, considérés comme les véhicules des idées dominantes et de la version officielle des événements, est de nos jours monnaie courante. Dans certaines pièces, ils deviennent une cible des auteurs, alors que des événements ou un autre thème sont les principaux objets de la critique. Ainsi que le donnent à voir les œuvres étudiées dans ce dossier, les médias apparaissent non seulement comme un facteur aggravant, mais comme un enjeu essentiel du drame, l'élément qui le suscite, l'embrayeur de la parole, la cause du conflit intérieur et de la scission du sujet, voire de sa possession, de sa dépossession ou de sa libération. À divers degrés, les discours des médias imprègnent dorénavant les dialogues, les monologues et même les didascalies, traces d'un monde envahissant dont il est devenu - pour le personnage et l'auteur dramatique - presque impossible de s'extraire sans faire, tel un nouveau misanthrope, le choix de renoncer au monde.

Le présent dossier offre donc un survol de la relation, souvent tendue, toujours critique, que la dramaturgie québécoise entretient avec les médias. Les auteurs convoqués appartiennent pour une part à la seconde moitié du $\mathrm{Xx}^{\mathrm{e}}$ siècle mais surtout au début du XXI ${ }^{e}$, moment où cette critique des médias a connu une vigueur et une étendue inégalées dans l'écriture dramatique. Si l'on remarque des absences (entre autres, Michel Tremblay, Jean-Pierre Ronfard, Normand Chaurette, Daniel Danis, François Archambault, Wajdi Mouawad, Pierre-Michel Tremblay) auxquelles il a fallu se résoudre, on notera en contrepartie une représentation abondante des jeunes auteurs aux côtés de figures clés de notre dramaturgie: Claude Gauvreau, Carole Fréchette, Larry Tremblay et Évelyne de la Chenelière. Le média dont il est le plus souvent question est la télévision, et les auteurs l'examinent sous plusieurs angles: que ce soit à titre de producteur d'information ou de fiction, qu'il s'agisse de la télévision publique ou des diffuseurs privés. Tantôt le pouvoir de l'image, des clichés et des stéréotypes ${ }^{18}$

17 «Engagement nouvelle vague», n 94, mars 2000; "Thêâtre d'intervention», n 113, décembre 2004; "Subversion", $n^{\circ}$ 135, juin 2010; "Jouer dans la cité», n 139, juin 2011. 18 Soulignons l'association métonymique étroite entre les inventions qui ont permis l'apparition des médias de masse et le principe esthétique de la répétition figée, banalisée : ces deux notions désignaient à l'origine des plaques d'impression destinées à produire 
est convoqué, tantôt la capacité des individus d'y résister, qui est incertaine et paradoxale ${ }^{19}$. La presse revient également dans plusieurs pièces. Internet commence à peine à faire partie du paysage dramatique : ses virtualités sont exposées et son usage croisé dans la société et dans l'écriture dramatique est noté. D'autres pièces mettent l'ensemble des médias dans le même sac, sans bien distinguer ce qui relève de la radio ou de la télévision, de la presse ou d'Internet. Ce qui compte ici, c'est l'effet de système, l'amplification du discours dominant et le degré de pénétration de la réalité auxquels donnent lieu la réunion de toutes ces plateformes, cette polyphonie convergente, cette prolifération des écrits, des écrans, des caméras, des micros, des ordinateurs, des tablettes, des téléphones et autres appareils par lesquels l'individu est cerné de toutes parts. La préoccupation à l'égard des médias exprimée par le théâtre québécois d'aujourd'hui, c'est d'abord cela : ne pas accepter l'état des médias tel qu'il est et exiger d'eux davantage que leur prestation actuelle. Le revers de cette orientation dramaturgique est qu'elle s'aventure peu à dépeindre des héros, des modèles animés par un idéalisme ou des utopies susceptibles d'ébranler, de réformer ou de subvertir l'ordre médiatique, ne serait-ce que pour un temps. Nul Docteur Stockmann ${ }^{20}$, dans la dramaturgie québécoise contemporaine, qui voie dans le journal un véhicule de progrès à mettre au service de la vérité, quitte à en payer le prix. Comme l'hydre ne saurait être terrassée, certains s'en désespèrent, mais la majorité préfère en rire, s'en moquer, montrer du doigt ses incohérences et ses faiblesses.

Le dossier s'ouvre sur l'entretien avec Carole Fréchette et sur l'article de MarieChristine Lesage et d'Audrey-Anne Cyr, qui discutent tous deux de l'importance d'Internet dans la dramaturgie d'aujourd'hui. Pour ces dernières, la dépendance à Internet, aux médias sociaux et à la technoculture en général dépeinte dans les dispositifs dramatiques d'Olivier Choinière et de Guillaume Corbeil constitue une critique incisive de la société de l'hyperconsommation. Elles remarquent cependant que la surmédiatisation actuelle de la culture ne fait pas qu'engendrer de la passivité et de la dépossession, elle conduit aussi les individus à moins subir le système médiatique puisqu'ils s'en servent afin de se mettre eux-mêmes en scène. Au total, les machines médiatiques demeurent globalement, aux yeux de Corbeil et de Choinière, des mécaniques aliénantes, ce que leur dramaturgie parvient à faire éprouver au spectateur en reproduisant sur scène leurs effets enjôleurs. Pour sa part, dans l'entretien qu'elle accorde à Hervé Guay, l'auteure de Je pense à Yu se montre concernée par l'état des médias, avec lesquels elle et ses personnages composent, dans les deux sens du terme. Elle insiste notamment sur le rôle clé des médias au chapitre de la transmission de

\section{$+++$}

un très grand nombre d'exemplaires des mêmes pages. Voir Ruth Amossy, Les idées reçues: sémiologie du stéréotype, Paris, Nathan, coll. «Le texte à l'œuvre», 1991, p. 25. 19 «La résistance aux médias est d'autant plus grande qu'on les croit tout-puissants, capables de modifier à volonté les opinions ou les comportements des gens ; à l'inverse, on ne leur oppose aucun contrepoison, quand on les imagine parfaitement inoffensifs. Là réside le mystère ou l'ironie du "pouvoir" des médias : il est d'autant plus grand qu' on le croit faible, d'autant plus restreint qu'on le croit illimité.» Francis Balle, Les médias, Paris, Presses universitaires de France, coll. «Que sais-je?», 2004, p. 95.20 Le docteur Stockmann est le héros d'Un ennemi du peuple de Henrik Ibsen. Cette pièce créée à Oslo en 1883 met en scène un homme qui se servira de la presse pour dénoncer un problème environnemental dans lequel est impliqué son propre frère, s'attirant ainsi la vindicte populaire. 
l'information internationale ( $" c^{\prime}$ est le monde extérieur qui vient à soi ${ }^{21}$ ») et en tant que stimulants pour la création («Les médias alimentent l'imaginaire comme tout le reste ${ }^{22}$.»). L'article de Francis Ducharme traite de la comédie Les aboyeurs de Michel Marc Bouchard, dans laquelle le dramaturge se livre à une véritable charge contre l'une des antennes régionales de la télévision publique. La lecture critique de Ducharme met en relief le caractère politique de l'œuvre, même s'il s'agit a priori d'un simple divertissement estival. Selon lui, Bouchard dépeint les dérives sensationnalistes et jovialistes d'une station de télévision régionale où débarquent deux arrivistes appelés à couvrir l'actualité de Villebleue, cité imaginaire d'une exaspérante tranquillité. Au passage, cette satire écorche également le peu de respect de la direction tant pour le mandat qui lui échoit que pour le public qu'elle dessert. Alors que l'information était au centre des Aboyeurs, c'est, entre autres, la qualité de la fiction télévisuelle et ses effets sur le téléspectateur qui préoccupent Larry Tremblay dans Téléroman. Hervé Guay y voit un jeu de rêves, tel que l'a défini Jean-Pierre Sarrazac, forme à laquelle le dramaturge recourt pour exprimer ses réticences - et son amusement - à l'égard de l'émission Piscine municipale dont ses héros, pitoyables danseurs, sont des admirateurs inconditionnels. Selon Guay, la singularité de cette satire est de porter tout autant sur les productions audiovisuelles bas de gamme que sur la danse contemporaine, dont l'auteur brosse un portrait drolatique. Tremblay propose là une solution originale au problème de l'opposition facile entre culture savante et culture populaire qui sous-tend bien des critiques des médias de masse. Hélène Jacques envisage la question sous l'angle des relations troubles qu'entretiennent littérature et médias. L'imposture d'Évelyne de la Chenelière lui fournit une situation dramatique idéale où deux figures d'auteurs s'opposent à la faveur du battage médiatique organisé autour de la sortie du nouveau roman d'une mère qui demande à son fils, plus télégénique qu'elle, d'endosser son rôle d'auteure. Les concepts de paratopie et de communitas, empruntés respectivement à Dominique Maingueneau et à Michel Biron, lui sont utiles pour comprendre le rôle joué par les médias dans la définition de la posture de l'écrivain au sein de la société québécoise. Julie Sermon étudie le texte et la mise en scène de Vu d'ici, spectacle de Christian Lapointe tiré du roman au même titre de Mathieu Arsenault. Ses analyses montrent d'abord comment le réquisitoire violent contre la télévision auquel se livre le protagoniste de Vu d'ici emprunte à la pensée de certains intellectuels, tels Pier Paolo Pasolini et Guy Debord, des explications au sujet du fonctionnement et des effets idéologiques de la télévision. Son article souligne également à quel point le discours médiatique, à la fois comme texte et comme spectacle, constitue ce qu'elle appelle une «supra-identité venant infiltrer la voix et le corps du locuteur ${ }^{23}$ ». Noële Racine clôt ce dossier avec Claude Gauvreau, dont elle analyse les écrits sur le théâtre dans la presse. Elle nous présente l'auteur de La charge de l'orignal épormyable comme plus préoccupé de réfléchir à ce que son théâtre pourrait être qu'à l'état des médias de son époque. Il n'en représente pas moins le critique dans ses

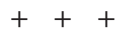

21 Voir Hervé Guay, "Entretien avec Carole Fréchette», dans le présent dossier, p. 19. 22 Ibid. 23 Voir Julie Sermon, «Tout contre la télévision. Didactique de l'adhérence et de la dénonciation dans Vu d'ici », dans le présent dossier, p. 87. 
pièces, conférant à l'Yvirnig des Oranges sont vertes une stature, un corps et un verbe qui confirment la haute idée que l'auteur de ce poème dramatique se faisait de cette profession, à condition que celui qui la pratique allie audace et liberté d'esprit.

L'ensemble du dossier prouve, en somme, qu'en dépit du préjugé défavorable de nombre d'auteurs à l'endroit de la culture médiatique, la rencontre entre thêâtre et médias s'avère féconde, tant pour la dramaturgie que pour la mise en scène. 